cleavage effects, pile-up round various surface hardness testing indentations, and machining surface quality. The title of the penultimate chapter, "Barton's Button", refers to a historical curiosity, some early 19th century metal buttons which wore cast with a coarse grating ruling pattern as a novelty for the dandies of the day; interferometry was used to determine the depth and profile of the rulings. Plastic moulded gratings hæve recently become available as ornaments on cufflinks, brooches, earrings, and as "skin jewels" for attaching to the forehead, arm cheek, and the like. Plus ca çhange ...

The book is beautifully produced, as one expcets from the publisher, and the half-tones of fringe patterns are superb. It can be recommended as an cxcellent addition to the bookshelf of anybody with an interest in interferometry.

W. T. WELFORD

\section{ATLAS OF THE HEAVENS}

\section{Smithsonian Astrophysical Observatory Star Atlas of} Reference Stars and Nonstellar Objects

Prepared by the staff of SAO. Pp. ix $+13+152$ star charts. (MIT Press: Cambridge, Massachusetts, and London, March 1970.) 173s.

ConsIDERING the wide range of uses for which charts are needed in optical astronomy, the number available is not excessive. The two most widely used classical atlases are the Bonner Durchmusterung or $B D$ catalogue and charts (with their southern visual and photographic extensions by the Cordoba and Cape Observatories respectively) and the Palomar Observatory--National Geographic Society Sky Survey, which has no general catalogue and is so rich that it is unlikely ever to acquire one. Astrographic catalogues and charts cover large zones of the sky to 14th magnitude (photographic) north of declination $-25^{\circ}$, but have coordinates inconveniently expressed in $x$ and $y$ and no data on galaxies and nebulre, while tho Franklin-Adams charts (with no catalogue) provide a poor man's sky survey that is still useful south of the Palomar survey's declination limit at $-33^{\circ}$. Other more widely available charts exist (for example, Norton's Star Atlas and the Skalnate Pleso atlases by A. Bečvar) and often provide a great deal of useful information, but they are limited either to fairly bright stars or to restricted zones of the sky.

Recent developments in astronomy and spacc research have increased the demand for rapid access to stars of accurately known position for optical tracking of solarsystem objects and space vehicles, for the proparation of finding charts and for the identification of radio, X-ray and infrared sources on the Palomar charts or on photographs taken with large telescopes. The $B D$-an extraordinary achievement in its time and still very useful after 100 years - is virtually complete to the 9 th magnitude and has many fainter stars as well, but it has many disadvantages. Its coordinates refer to the epoch of 1855 and often are inaccurate by one or two are minutes-in some cases even more. Its format is also unsuitable for use at the telescope. At large observatories, the tendency nowadays is to prepare appropriate charts or overlays for an interesting region by computer, using standard catalogues in machine-readable form. But, naturally, these facilities are not always available, and even when they are they may not be usable at short notice, for cxample, when one is suddenly asked to observe an Apollo vehicle that is getting into difficulties. Consequently the production of a good modern set of charts covering the whole sky, like the present $S A O$ Star Atlas, is a very welcome development.

This atlas is designed to be used in conjunction with the SAO Star Catalog (1966), which gives accurate positions (epoch 1950.0) and proper motions for some 250,000 stars and is almost complete to the 9 th magnitude.
(Some fainter stars are also included, but much fewer than in the $B D$.) Thus every star in the atlas (apart from about 1,000 additional stars of unknown proper motion in the South Polar Cap) has a precise position available and so is automatically suitable as a reference star for natural and man-made objects in the solar system or for the preparation of overlays for the Palomar charts and other photographs. In addition, galaxies (with the correct size and orientation), globular clusters, planetary nobulae and NGC objects of other categories are shown, denoted by special symbols. The scale is $0.863 \mathrm{~cm}$ per degree, matching that of photographs taken with the Baker-Nunn cameras, and transparent interpolation réseaux enable positions to be read off directly to an accuracy of about one arc minute. Prepared from the Catalog with tho aid of an analogue computer, the Atlas is divided into 15211 inch $\times 14$ inch eharts on stiff paper which can easily be handled at the telescope. This convenience is paid for to some extent by the rather small scale, which makes it advisable to use a magnifying glass, especially in a poorly lighted dome.

At just under $1 s 2 d$ per chart, this excellent atlas is very reasonably priced and it can be most highly recommended to amateur and professional astronomers alike.

Bernard Pagel

\section{DECISION MAKING}

Decision Theory

By D. J. White. (The Centre for Business Research, University of Manchester.) Pp. ix +185 . (Allen and Unwin: London, November 1969.) $48 s$.

THrs book is essentially a report of the findings of a research project carried out by the author while at the Manchester Business School. The project was concerned with the content of decision theory and its importance in practical decision making. The chief emphasis is placed on an examination of the various conccpts which have been introduced in studying the problem of decision. It must be said that the whole subject is only at an interim stage in its development, and there scem to be very few firm conclusions.

A long chapter is devoted to theories of choice, value and uncertainty. Then, after a chapter on decidability, there are some practical considerations in decision analysis. These include a comparison between the physical and human sciences, and it is at this point particularly that one realizes that the subject matter of decision theory consists of a subtle complicated area of human thought which is, at the same time, the concern of the physical scientist, the behavioural scientist, the logician and the philosopher. After a chapter on pragmatic aspects of decision theory, the book concludes with a chapter on mathematical models and decision.

It must be said that the author has written what, to many people, will appear as a learned account of a difficult subject. If anybody hopes that his personal powers of decision making might be improved by reading the book, he is likely to be disappointed. There is no gentle introduction to the subject matter; and, indeed, the first sentence in the text occupies no less than seven lines of print. The reader, if he is to understand the account of value, will have to be able to think of value as an order preserving homomorphism, and to know what is meant by a boolean algebra of sets. Also, in order to understand the discussion of uncertainty the reader will need some knowledge and appreciation of the Heisenberg principle of uncertainty, as expounded in the theory of quantum mechanics. All this is very exciting, but it is only for real scholars, with considerable background knowledgein diverse fields, and with real mental span. It is in the nature of the subject, and the author is to be congratulated on the masterly way in which he has bridged the void between the physical and human aspects of the subject. L. S. GoDDARD 